\title{
Aplicación de quitosano como biocoagulante en aguas residuales contaminadas con hidrocarburos
}

\section{(Chitosan application as a biocoagulant in wastewater contaminated with hydrocarbons)}

\author{
Juan M. Álava ${ }^{1}$
}

\begin{abstract}
Resumen:
La contaminación del ambiente en el Ecuador, provocada por la producción, transporte y comercialización de hidrocarburos, requiere mayor investigación en cuanto a nuevas alternativas de tratamiento que utilicen sustancias biodegradables. En este estudio, se utilizó el exoesqueleto de la cola del camarón (Litopenaeus vannamei) para obtener quitosano y luego aplicarlo como biocoagulante a una muestra de agua residual contaminada con residuos hidrocarburíferos. El quitosano producido fue caracterizado por titulación potenciométrica, resultando en un grado de desacetilación (\%DD) de $87.18 \%-93.72 \%$ y por viscosimetría intrínseca, logrando un peso molecular promedio $(\mathrm{g} / \mathrm{mol})$ de $5.2 \times 10^{5}-5.4 \times 10^{5}$. La aplicación del quitosano se la realizó en un test de jarras, para lo cual se planteó un diseño factorial completamente aleatorio $2^{\mathrm{k}}$, resultando en un efecto estadísticamente significativo para todos los factores estudiados, esto es, $\mathrm{pH}$ (inicial), tipo de quitosano y método de agitación, utilizando como variable de respuesta al porcentaje de remoción de turbidez. Como resultado, un pH de 5.5 , una dosis de $2 \mathrm{mg}$ (quitosano)/L(muestra) y método de agitación rápido fueron aplicados a una muestra contaminada, disminuyendo la turbidez en $98.19 \%$, la demanda química de oxígeno en $78.17 \%$, el color en un $91.45 \%$ e hidrocarburos totales de petróleo en $99.09 \%$.
\end{abstract}

Palabras clave: Test de jarras; exoesqueleto de camarón; clarificación; desacetilación; viscosimetría intrínseca.

\begin{abstract}
:
The environment contamination in Ecuador, done by the production, transport and commercialization of hydrocarbons, requires further research regarding new treatment alternatives that use biodegradable substances. In this study, abdominal shrimp shell waste, Litopenaeus vannamei was used to obtain chitosan and then apply it as a biocoagulant to a wastewater sample contaminated with hydrocarbon products. The produced chitosan was characterised by potentiometric titration, resulting in a deacetylation degree (\%DD) of $87.18 \%-$ $93.72 \%$ and by intrinsic viscosimetry, obtaining an average molecular weight $(\mathrm{g} / \mathrm{mol})$ of $5.2 \times 10^{5}$ $-5.4 \times 10^{5}$. The application of chitosan was done in a jar test, for which a completely randomised factorial design $2^{\mathrm{k}}$ was set, resulting in an evident statistically significant effect for all the factor studied, that is, $\mathrm{pH}$ (Initial), chitosan type and agitation method, using the turbidity percentage removal as the response variable. As a result, a pH of 5.5, a $2 \mathrm{mg}$ (Chitosan)/L(sample) and a fast agitation method were applied to a contaminated sample reducing the turbidity in $98.19 \%$, the oxygen chemical demand in $78.17 \%$, color in $91.45 \%$ and total petroleum hydrocarbon in $99.09 \%$.
\end{abstract}

Keywords: Jar test; shrimp shell; clarification; deacetylation; intrinsic viscosimetry.

\footnotetext{
${ }^{1}$ Universidad Técnica de Manabí, Portoviejo - Ecuador (jalava4502@utm.edu.ec)
} 


\section{Introducción}

La contaminación del suelo y principalmente del agua a nivel global por hidrocarburos ha dejado consecuencias negativas para el medioambiente. En ciertos casos de derrames de hidrocarburos, como en West Falmouth, los sedimentos muestran que luego de más de 30 años la presencia de hidrocarburos continúa y continuará indefinidamente (Reddy et al., 2002). El Ecuador, siendo un país petrolero no está alejado de esta realidad y varios incidentes han sido reportados en las diferentes etapas de procesamiento y comercialización del mismo, en donde se muestra el nivel de contaminación que ha dejado como por ejemplo en la costa Ecuatoriana (5-50 mg/l TPH) y que en algunos casos se mantiene presente (Da Ros, 1995). En base a esta problemática, a nivel mundial se han desarrollado mecanismos de remediación de hidrocarburos tanto físicos, químicos electroquímicos, bilógicos, entre otros; siendo el uso de biocoagulantes como el quitosano una alternativa efectiva para remover hidrocarburos y demás contaminantes en los cuerpos de agua producto de la actividad petrolera y diferentes actividades industriales, debido a sus características biodegradables, no tóxicas y biocompatibles (Rodríguez, 2011); (Caldera et al., 2009); (Renault et al., 2009); (Abu Hassan, 2009); (Sastre, Ruiz, Guibal, \& Szygula, 2010).

Además del valor que representa el camarón como alimento, el residuo del camarón también contiene, entre otros componentes, alrededor de $52 \%$ de proteína, $24 \%$ de minerales y $18 \%$ de quitina, los cuales han sido desechados en el Ecuador, en su mayoría sin darle valor agregado. La quitina es el elemento más abundante en la naturaleza después de la celulosa y el elemento de sostén de los invertebrados y ha sido investigada por más de un siglo, obteniéndose muchos usos que le dan valor agregado como la obtención de quitosano por desacetilación de la quitina (Agulló et al., 2004). En este sentido, el Ecuador es uno de los principales productores de camarón en el mundo ocupando el séptimo lugar y a nivel regional es el primero (Varela, 2011), representando el 25.19\% de toda América Latina. Adicionalmente, para el 2013 existió una evolución destacada de las exportaciones con un aumento del $25.63 \%$, pasando de $\$ 443,3$ millones a 565,8 millones (Banco Central del Ecuador, 2014).

El objetivo de este estudio es poder determinar el efecto que tiene el quitosano, $\mathrm{pH}$ y método de agitación en el proceso de clarificación de aguas contaminadas con hidrocarburos, además de demostrar la capacidad de remoción del quitosano como agente biocoagulante bajo las mejores condiciones de aplicación a una muestra con altos niveles de contaminación.

\section{Metodología}

\subsection{Obtención de quitosano}

En el proceso de obtención se utilizó el residuo del empacado de camarón facilitado por la empresa Dufer ubicada en Bahía de Caráquez en la costa Ecuatoriana. El exoesqueleto de cola de camarón fue procesado de acuerdo al diagrama de la Figura 1. con el objeto de remover 
proteínas, minerales, pigmentos y demás impurezas por medios químicos para obtener como producto final la quitina, mediante el método propuesto por Pinelli Saavedra, Toledo Guillen, Esquerra Brauer, Luviano Silva, and Higuera Ciapara (1998). Seguidamente, se utilizaron etapas sucesivas para desacetilar la quitina con $\mathrm{NaOH} 50 \% \mathrm{p} / \mathrm{p} \quad 90^{\circ} \mathrm{C}$ por $1.5 \mathrm{~h}$ a una proporción solvente:soluto de 50:1 v/p en agitación constante de acuerdo a Yaghobi and Mirzadeh (2004) en presencia de aire atmosférico.

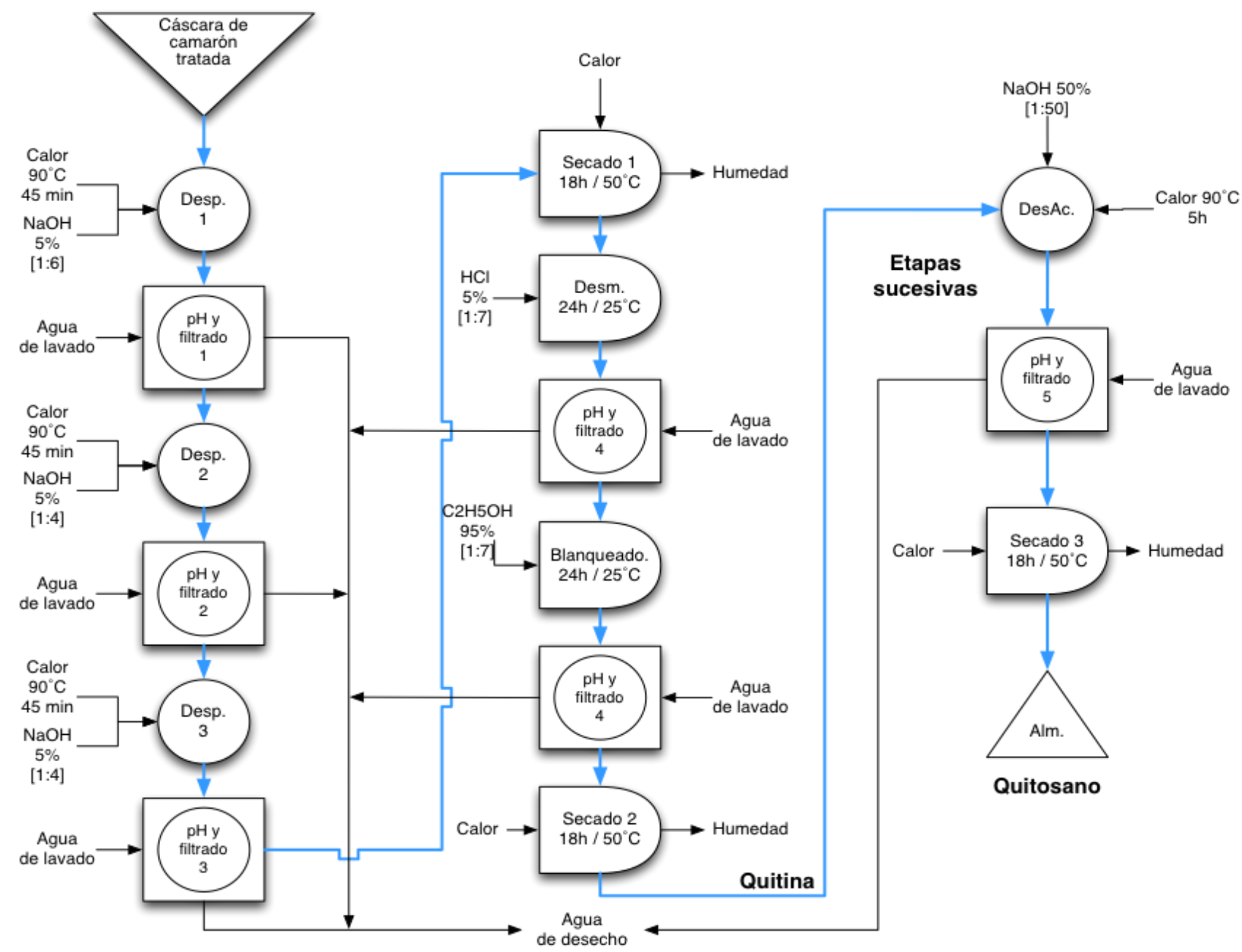

Figura 1. Proceso químico de obtención de quitina y quitosano utilizando etapas sucesivas.

\subsection{Caracterización de quitosano}

\subsubsection{Peso molecular promedio (M)}

Se utilizó el método viscosimétrico, en donde se empleó un viscosímetro capilar Cannon-Fenske 150 Y137 (ASTM D445) con baño termostático a $25^{\circ} \mathrm{C}$. En el viscosímetro se corrieron las muestras de quitosano disueltas en ácido acético $0,1 \mathrm{M}$ y cloruro de sodio $0.2 \mathrm{M}$ con diferentes concentraciones, determinando el tiempo de caída de las diluciones de quitosano ( $\mathrm{t}$ ) y una de referencia con nula concentración ( $t_{0}$ ) (Paz et al., 2013) para determinar los parámetros viscosimétricos como se establece en la Tabla 1. 
Tabla 1. Datos viscosimétricos utilizados en la determinación del peso molecular promedio por viscosimetría intrínseca.

\begin{tabular}{|c|c|}
\hline Nombre común & Símbolo y ecuación \\
\hline Viscosidad relativa & $\eta_{\mathrm{r}}=\eta / \eta_{0}=\mathrm{t} / \mathrm{t}_{0}$ \\
\hline Viscosidad específica & $\eta_{\mathrm{sp}}=\eta_{\mathrm{r}}-1=\left(\eta-\eta_{0}\right) / \eta_{0}=\mathrm{t}-\mathrm{t}_{0} / \mathrm{t}_{0}$ \\
\hline Viscosidad reducida & $\eta_{\mathrm{red}}=\eta_{\mathrm{sp}} / \mathrm{C}$ \\
\hline Viscosidad inherente & $\eta_{\mathrm{red}}=\left(\operatorname{In} \eta_{\mathrm{r}}\right) / \mathrm{C}$ \\
\hline Viscosidad intrínseca & {$[\eta]=\left(\eta_{\mathrm{sp}} / \mathrm{C}\right)_{\mathrm{C}=0}=\left[\left(\ln \eta_{\mathrm{red}}\right) / \mathrm{C}\right]_{\mathrm{C}=0}$} \\
\hline
\end{tabular}

Los parámetros citados se emplearon para la determinación de la viscosidad intrínseca [ $\eta$ ] mediante el método gráfico, empleando la ecuación de Huggings (Ec. 1).

$$
\eta_{s p} / C=[\eta]+K[\eta]^{2} C
$$

Esta fórmula relaciona la viscosidad reducida $\left(\eta_{\mathrm{sp}} / \mathrm{C}\right)$ con la concentración del polímero y se utiliza para determinar el peso molecular promedio (M) de acuerdo a la ecuación de Mark-HouwinkSakurada (Ec. 2).

$$
\eta=K M^{a}
$$

En donde las constantes de la ecuación para tal medio son: $\mathrm{K}=1.81 \times 10^{-3} \mathrm{~cm}^{3} \mathrm{~g}$ y $\alpha=0.93$ de acuerdo a Roberts and Domszy (1982)

\subsubsection{Grado de desacetilación \%DD}

Para el ensayo se disolvieron $0.2 \mathrm{~g}$ de quitosano seco en $20 \mathrm{~cm}^{3}$ de ácido clorhídrico $0.1 \mathrm{M}$ y 25 $\mathrm{cm}^{3}$ de agua desionizada, se los dejó en agitación continua por 30 minutos y luego se agregó 25 $\mathrm{cm}^{3}$ más de agua y se agitó por 30 minutos más. Una vez que el quitosano se disolvió completamente se procedió a titular la solución con hidróxido de sodio, con una concentración de $0.1 \mathrm{M}$, utilizando una micropipeta Eppendorf (1000 $\mu \mathrm{l}$ ) añadiendo $500 \mu \mathrm{l}$ de $\mathrm{NaOH}$ cada vez y anotando el cambio en el pH con un potenciómetro con electrodo de vidrio marca OAKTON pH 700. Una vez alcanzado un $\mathrm{pH}$ de alrededor de 11, se tabularon los resultados en una hoja de cálculo para ver el comportamiento del $\mathrm{pH}$ y su derivada en función del volumen de $\mathrm{NaOH}$ utilizado; mediante este gráfico se determinaron los puntos de inflexión ( $V_{1}$ y $\left.V_{2}\right)$ (CzechowskaBiskup, Jarosińska, Rokita, Ulański, \& Rosiak, 2012). El grado de desacetilación \%DD se calculó usando la fórmula:

$$
\% \mathrm{DD}=2.03 \cdot[(\mathrm{V} 1-\mathrm{V} 2) /(\mathrm{m}+0.0042 \cdot(\mathrm{V} 2-\mathrm{V} 1)]
$$

En donde:

$\mathrm{m}=$ masa de la muestra $(\mathrm{g})$

V1, V2 = Volúmenes de la solución de hidróxido de sodio $0.1 \mathrm{~mol} / \mathrm{dm}^{3}$ correspondientes a los puntos de deflexión. 
2.03 = Es el coeficiente resultante del peso molecular de la unidad de monómero de la quitina.

0.0042 = Coeficiente resultante de la diferencia entre el peso molecular de la unidades de los monómeros de quitina y quitosano.

\subsection{Caracterización del agua contaminada con derivados petroleros}

Las muestras de agua contaminada fueron proporcionadas por una estación de servicio de la ciudad de Portoviejo en Manabí. Para el diseño experimental se recogió muestra suficiente para llevar a cabo los 32 experimentos. Las muestras se tomaron de la primera de las tres trampas de grasa que recolecta los residuos líquidos resultantes del expendio de los derivados del petróleo antes de ser eliminada por los gestores ambientales. En lo que respecta al procedimiento de toma de muestras, para realizar análisis de TPH en el laboratorio acreditado, se siguió la norma NTE INEN 2 169:98 (Agua, calidad de agua, muestreo, manejo y conservación de muestras). Adicionalmente, en el laboratorio de Aguas y Medio Ambiente de la Universidad Técnica de Manabí se realizaron las pruebas de: turbidez (NTU) en un turbidímetro marca $\mathrm{HACH}$, demanda química de oxígeno DQO (mg/l)(Método del dicromato de potasio) y color (Unidades PtCo)(Método estándar Platino-Cobalto) en un espectrofotómetro UV-Vis marca HACH 2700. Adicionalmente, se procedió a enviar muestra suficiente al laboratorio acreditado Grupo Químico Marcos para la determinación de Hidrocarburos Totales de Petróleo, Cromo, Vanadio, Bario y Plomo.

\subsection{Aplicación de quitosano en la muestra contaminada}

\subsubsection{Preparación de soluciones de quitosano}

El quitosano obtenido fue disuelto en ácido acético $0.1 \mathrm{M}$ en agitación constante por $24 \mathrm{~h}$ a temperatura ambiente para obtener una solución de quitosano al 0,5\% aproximadamente, la cual fue filtrada con el propósito de eliminar cualquier material insoluble (Paz et al., 2012). El quitosano se aplicó con micropipetas marca Eppendorf (1000 $\mu \mathrm{l}, 200 \mu \mathrm{l}$ y $20 \mu \mathrm{l})$ en la prueba de jarras.

\subsubsection{Proceso de clarificación de muestras contaminadas}

Este proceso consta de dos etapas: primero, se planteó un diseño experimental (DOE) utilizando una muestra (Mc1) con el objeto de determinar las variables que influyen en el proceso de clarificación y a su vez establecer un modelo de comportamiento estadístico de las variables evaluadas en función de el porcentaje de remoción de turbiedad. Seguidamente, estos resultados se emplearon para clarificar una muestra con altos niveles de contaminación (Mc2) la cual fue caracterizada antes y después del tratamiento en turbiedad, DQO, color, TPH y metales pesados.

\subsubsection{Planteamiento del diseño experimental}

Se planteó un diseño experimental completo, aleatorio, de dos niveles (Tabla 2), con el objeto de 
evaluar el efecto que tienen: el pH de la muestra, el tipo de quitosano empleado y el método de agitación del test de jarras en el proceso de clarificación de la muestra utilizada. Este proceso se lo realizó en un test de jarras, teniendo como variable de respuesta al porcentaje de remoción de turbiedad para un volumen de muestra de $1 \mathrm{~L}$.

Tabla 2. Factores evaluados para el proceso de clarificación de la muestra Mc1.

\begin{tabular}{|c|c|c|c|c|c|}
\hline Factores & $\begin{array}{c}\text { Nivel } \\
-\end{array}$ & $\begin{array}{c}\text { Nivel } \\
+\end{array}$ & Réplicas & $\begin{array}{c}\text { Tratamientos } \\
\text { Totales }\end{array}$ & Variable de respuesta \\
\cline { 1 - 3 } $\mathrm{pH}(\mathrm{X} 1)$ & 5.5 & 7.5 & \multirow{2}{*}{3} & 32 & $\begin{array}{c}\text { Porcentaje de remoción } \\
\text { de turbidez \% }\end{array}$ \\
\cline { 1 - 3 } Tipo de Quitosano (X2) & $\mathrm{Q} 1$ & $\mathrm{Q} 2$ & & & \\
\hline Método de agitación (X3) & $\mathrm{M} 1$ & $\mathrm{M} 2$ & & & \\
\hline
\end{tabular}

Tanto el diseño experimental como el análisis estadístico fueron desarrollados en el paquete estadístico JMP (9.0.1; 2010 SAS Institute Inc.). En la Tabla 3 se muestra el patrón de diseño a seguir, establecido por el programa con los respectivos niveles de variación.

Tabla 3. Patrón de diseño experimental, evaluación de factores en el proceso de clarificación

\begin{tabular}{|c|c|c|c|}
\hline $\begin{array}{c}\text { Patrón de } \\
\text { tratamiento }\end{array}$ & $\mathbf{p H}\left(\mathbf{X}_{\mathbf{1}}\right)$ & $\begin{array}{c}\text { Tipo de Quitosano } \\
\left(\mathbf{X}_{\mathbf{2}}\right)\end{array}$ & $\begin{array}{c}\text { Método de Agitación } \\
\left(\mathbf{X}_{\mathbf{3}}\right)\end{array}$ \\
\hline$+\mathbf{1 1}$ & 7.5 & $\mathrm{Q} 1$ & $\mathrm{M} 1$ \\
\hline+12 & 7.5 & $\mathrm{Q} 1$ & $\mathrm{M} 2$ \\
\hline+21 & 7.5 & $\mathrm{Q} 2$ & $\mathrm{M} 1$ \\
\hline+22 & 7.5 & $\mathrm{Q} 2$ & $\mathrm{M} 2$ \\
\hline-11 & 5.5 & $\mathrm{Q} 1$ & $\mathrm{M} 1$ \\
\hline-12 & 5.5 & $\mathrm{Q} 1$ & $\mathrm{M} 2$ \\
\hline-21 & 5.5 & $\mathrm{Q} 2$ & $\mathrm{M} 1$ \\
\hline-22 & 5.5 & $\mathrm{Q} 2$ & $\mathrm{M} 2$ \\
\hline
\end{tabular}

Donde el método de agitación M1 se dio en las etapas rápida a 250 rpm por 5 min. y lenta a 40 rpm por $20 \mathrm{~min}$. con un tiempo de reposo de $30 \mathrm{~min}$. Mientras que el método de agitación M2 tuvo una etapa rápida a $150 \mathrm{rpm}$ por $7 \mathrm{~min}$. y lenta a $25 \mathrm{rpm}$ por $30 \mathrm{~min}$. con un tiempo de reposo de 30 $\min$.

\subsubsection{Determinación de la dosis óptima de quitosano}

Se utilizó un equipo de Prueba de Jarras marca Phipps \& Bird de cuatro posiciones para llevar a cabo el proceso de clarificación de la muestra contaminada. Inicialmente, se probaron concentraciones de $0.2,0.4,0.6$ y $0.8 \mathrm{mg} / \mathrm{L}$, para la muestra Mc1, obteniéndose el mejor resultado para la dosis de $0.8 \mathrm{mg} / \mathrm{L}$. Mientras que para la muestra Mc2 las dosis probadas fueron de $0.5,1$, 1.5 y $2 \mathrm{mg} / \mathrm{L}$ resultando la dosis de $2 \mathrm{mg} / \mathrm{L}$ la mejor dosis.

\subsubsection{Tratamiento de agua residual contaminada con derivados del petróleo}

Se caracterizó la muestra Mc2 antes y después del tratamiento en el laboratorio de Aguas y Medioambiente de la UTM y enviando muestras a el laboratorio acreditado Grupo Químico Marcos. Utilizando los resultados del análisis estadístico, se plantearon las mejores condiciones 
para el proceso de clarificación de esta muestra, es decir, $\mathrm{pH} 5.5$, quitosano Q2 y método de agitación M1. El proceso se lo realizó en el test de jarras por triplicado utilizando un volumen de $2 \mathrm{~L}$ y una concentración de quitosano de 2 mg/L como se explica en la Figura 2.

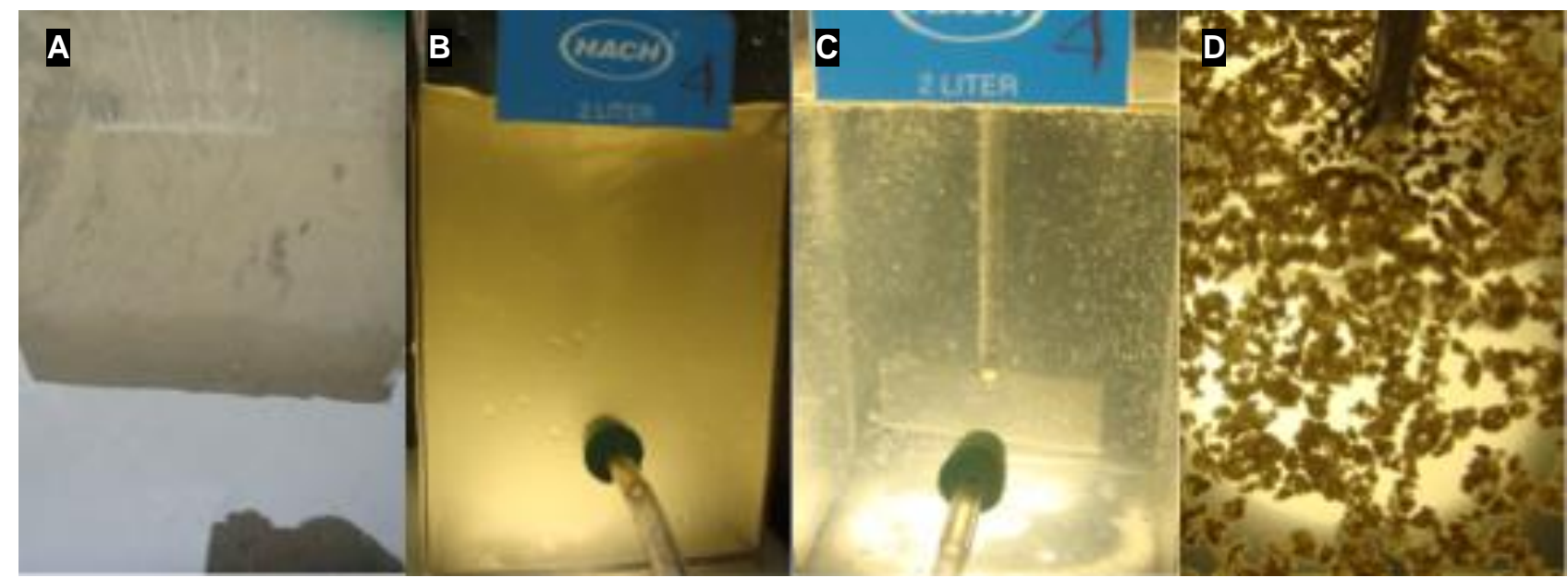

Figura 2. Proceso de clarificación de la muestra Mc2 contaminada con derivados del petróleo. En donde A revela el estado de la muestra desde la fuente de origen, $\mathbf{B}$ el nivel cualitativo de contaminación antes del tratamiento con quitosano, $\mathbf{C}$ clarificación de la muestra contaminada después del tratamiento y $\mathbf{D}$ se observan los flóculos flotantes formados en el proceso de clarificación.

\section{Resultados}

\subsection{Rendimientos en la obtención de quitosano}

La Figura 3 representa las cantidades promedio extraídas en masa de proteínas $53.1 \%$ y minerales $24.5 \%$ además de destacar el contenido de quitina $21.1 \%$ y de quitosano $16.1 \%$ con respecto a la materia prima utilizada y en comparación con lo establecido por Agulló et al. (2004).

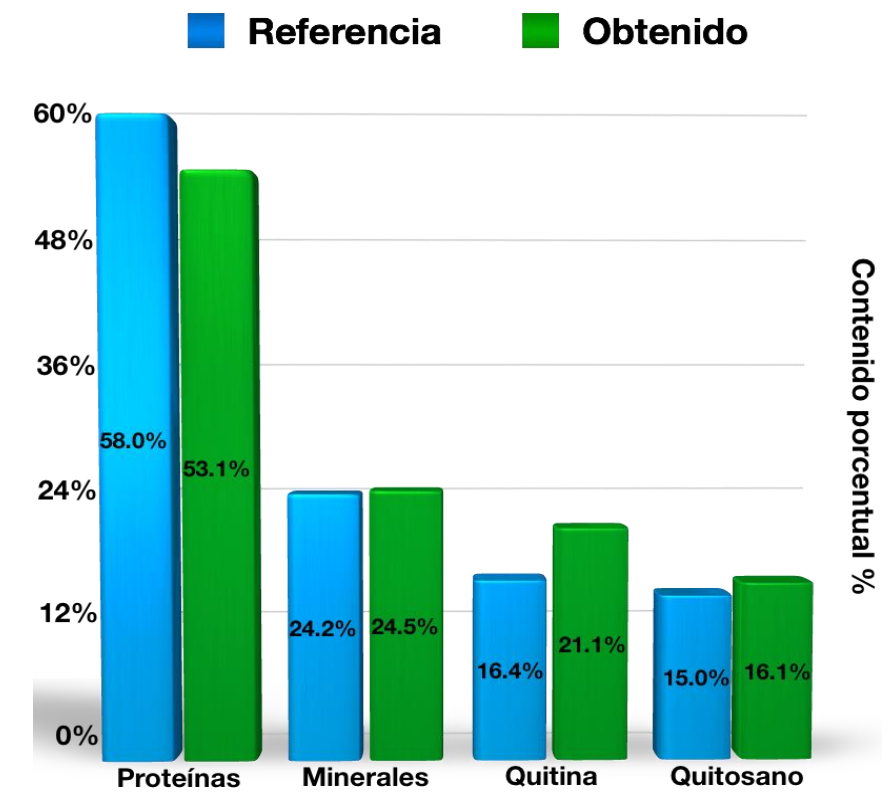

Figura 3. Contenido másico porcentual de proteínas, minerales, quitina y quitosano presentes en la muestra de cáscara de cola de camarón estudiada (base seca). 


\subsection{Caracterización del quitosano obtenido}

En la Tabla 4 se muestran los datos obtenidos para calcular el porcentaje de desacetilación (\%DD) por el método de titulación potenciométrica y el peso molecular promedio (Mv) por viscosimetría intrínseca, para los dos tipos de quitosano Q1 y Q2.

Tabla 4. Datos de ensayos de caracterización del quitosano \%DD y Mv.

\begin{tabular}{|c|c|c|c|}
\hline Ensayo & Unidades & Q1 & Q2 \\
\hline Titulación Potenciométrica $^{1}$ & \%DD & $87.18 \%$ & $93.72 \%$ \\
\hline Masa de quitosano & $g$ & 0.2004 & 0.2008 \\
\hline Volumen de inflección V1 & $\mathrm{ml}$ & 10 & 10 \\
\hline Volumen de inflección V2 & $\mathrm{ml}$ & 20.5 & 21.5 \\
\hline Número de Viscosidad Limitante (LVN) ${ }^{2}$ & Mv (g/mol) & $5.2 E+05$ & $5.4 \mathrm{E}+05$ \\
\hline Viscosidad Intrínseca & $\mathrm{ml} / \mathrm{g}$ & 377.9 & 391.25 \\
\hline Valor de la pendiente & & 58818 & 56392 \\
\hline Constante K & & 0.4 & 0.3 \\
\hline $\mathrm{R}^{2}$ & & 0.9813 & 0.9748 \\
\hline
\end{tabular}

\subsection{Tratamiento de la muestra contaminada con quitosano}

\subsubsection{Remoción de turbidez}

Los resultados del diseño experimental se presentan en la Figura 4, en donde la turbiedad final está en NTU para cada patrón de tratamiento con sus respectivas réplicas, para una muestra Mc1 con 80.3 NTU de turbidez.

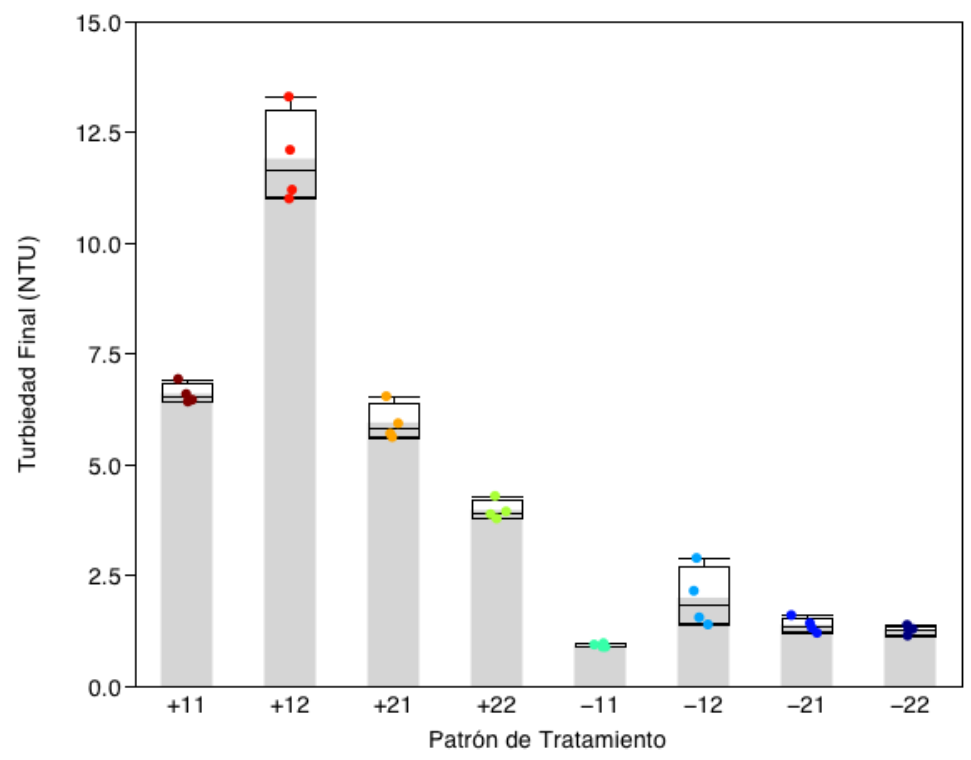

Figura 4. Turbidez final y dispersión de los resultados en función del patrón de tratamiento, destacando (-11) con menor turbidez final donde "-" es (pH 5.5), "1" (Q1) y "1" (M1). 


\subsubsection{Ajuste por mínimos cuadrados}

El modelo de comportamiento de las variables estudiadas (Figura 5) presenta una correlación lineal con un $\mathrm{R}^{2}=0.94$ y un factor de probabilidad de $\mathrm{P}<0.0001$ lo que indica que el modelo se ajusta de buena manera y que por lo menos existe un factor de regresión estadísticamente significante para dicho modelo.

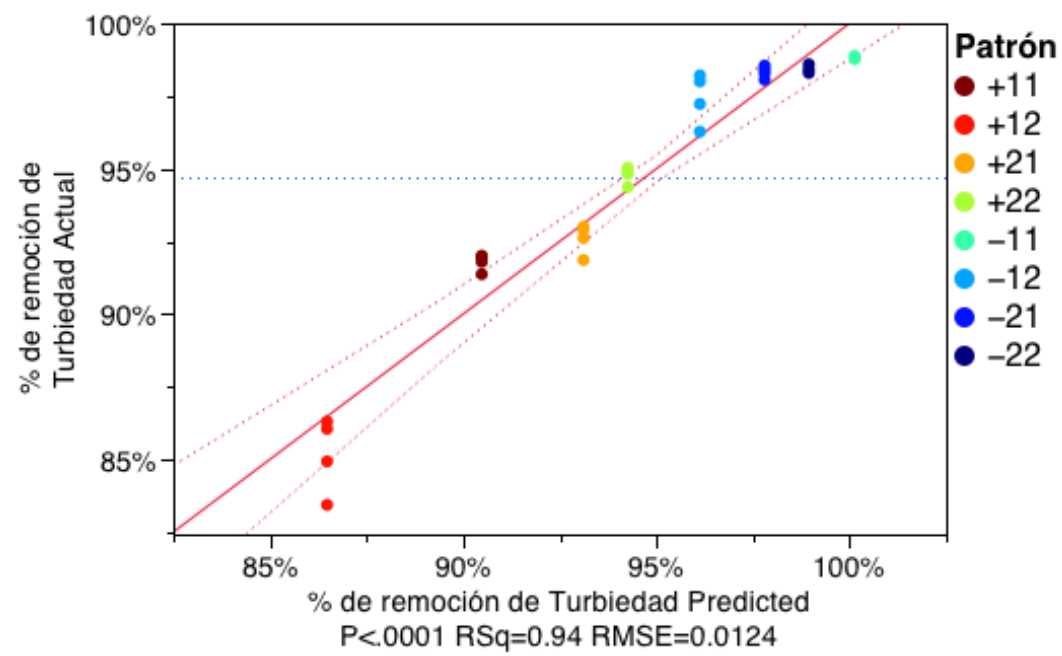

Figura 5. Ajuste por mínimos cuadrados para datos reales vs. Modelado para cada patrón de tratamiento.

Seguidamente, en la Tabla 5 se presenta la estimación de parámetros de probabilidad para cada uno de los factores estudiados y sus principales interacciones para los cuales se puede notar un factor de probabilidad $\mathrm{P}<0.0001$ principalmente para el $\mathrm{pH}$, quitosano, y uno de $\mathrm{P}<0.0032$ para el método de agitación, lo que indica el rechazo de la hipótesis nula y la aceptación de que existe una diferencia estadísticamente significativa entre los niveles de los factores estudiados ya que todos los valores se encuentran por debajo del $\mathrm{P}<0.05$.

Tabla 5. Estimación de parámetros de modelado con análisis de probabilidad

\begin{tabular}{|c|c|c|c|c|}
\hline Término & Estimado & Std Error & t Ratio & Prob $>|\mathbf{t}|$ \\
\hline Intersección & 0.9466741 & 0.0021990 & 430.4200000 & $<.0001^{*}$ \\
\hline Ph (5.5-7.5) & -0.035837 & 0.0021990 & -16.29 & $<.0001^{*}$ \\
\hline Quitosano [Q1] & -0.013628 & 0.0021990 & -6.2 & $<.0001^{*}$ \\
\hline Método de agitación [M1] & 0.0071456 & 0.0021990 & 3.25 & $0.0032^{*}$ \\
\hline $\mathrm{pH}^{\star}$ Quitosano [Q1] & -0.012402 & 0.0021990 & -5.64 & $<.0001^{*}$ \\
\hline $\begin{array}{l}\text { Quitosano [Q1] Método de agitación } \\
\text { [M1] }\end{array}$ & 0.0128691 & 0.0021990 & 5.85 & $<.0001^{*}$ \\
\hline $\begin{array}{l}\text { Probabilidad del parámetro de tener efecto nulo o cero. Se rechaza la hipótesis nula y se acepta la } \\
\text { alternativa. }\end{array}$ \\
\hline
\end{tabular}

\subsubsection{Tratamiento de muestra contaminada Mc2}

Se probó el quitosano en una muestra, con niveles altos de contaminación Mc2 (proporcionada por una estación de servicio para fines de la investigación) y con un volumen mayor (2 L) que el utilizado en el diseño experimental $(1 \mathrm{~L})$. Los análisis realizados a la muestra antes y después del 
tratamiento de clarificación con quitosano se presentan en la Tabla 6, en donde se muestra el contenido indetectable de Bario, Cromo, Plomo, Vanadio, de acuerdo al laboratorio acreditado.

Tabla 6. Porcentaje de remoción de contaminantes.

\begin{tabular}{|c|c|c|c|}
\hline Parámetro* & Valor Inicial & Valor Final & \% Removido \\
\hline Turbidez (NTU) & 216 & 3.92 & $98.19 \%$ \\
\hline DQO (mg/L) & 568 & 124 & $78.17 \%$ \\
\hline $\mathrm{pH}$ & 8.17 & 5.51 & Adj. ${ }^{1}$ \\
\hline Color (Units) & 655 & 56 & $91.45 \%$ \\
\hline TPH (mg/L) & 416 & 3.80 & $99.09 \%$ \\
\hline Bario (mg/L) & $<0.1$ & - & - \\
\hline Cromo (mg/L) & $<0.01$ & - & - \\
\hline Plomo (mg/L) & $<0.05$ & - & - \\
\hline Vanadio (mg/L) & $<0.05$ & - & - \\
\hline \multicolumn{4}{|c|}{$\begin{array}{l}\text { Análisis realizados en la Universidad Técnica de Manabí y por el laboratorio Grupo Químico } \\
\text { Marcos. } \\
1 \text { El pH fue ajustado para lograr los mayores porcentajes de remoción de turbidez de acuerdo } \\
\text { al modelo estadístico obtenido. }\end{array}$} \\
\hline
\end{tabular}
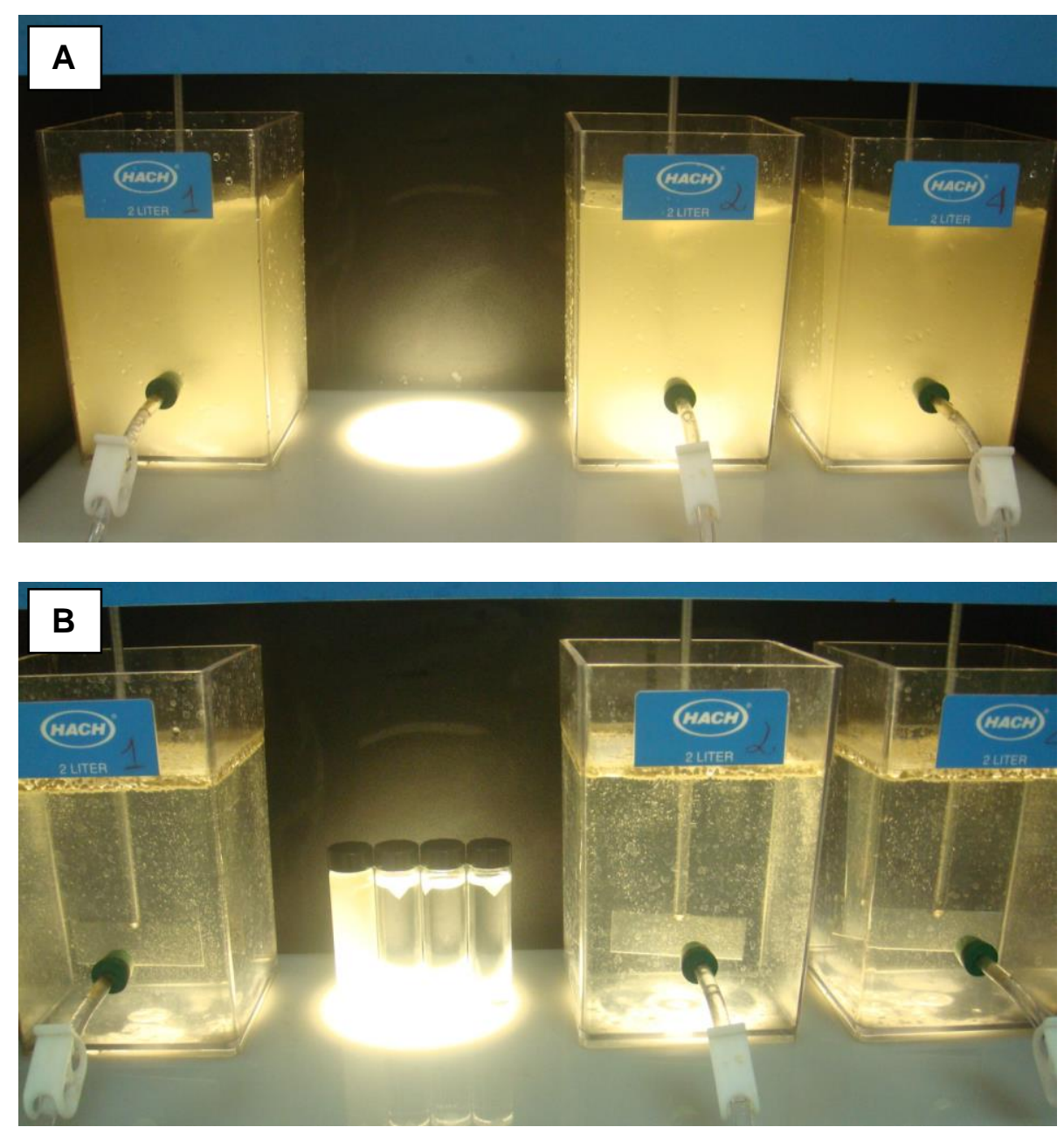

Figura 6. Test de jarras aplicando ph 5.5, quitosano Q1 y método de agitación M1 para la muestra Mc2. A Muestra contaminada con derivados petroleros.

B Muestra tratada con quitosano usando el modelo estadístico. 


\section{Discusión}

Con respecto a la obtención del quitosano, en la Figura 3 se presentan los valores promedio de componentes existentes en la muestra de exoesqueleto de camarón estudiada; ya que en el proceso de desproteinización se removió un 50\% de materia para Q1 y 56\% para Q2; mientras que la pérdida de masa desde la desproteinización hasta terminar la desmineralización fue de $26 \%$ para Q1 y $23 \%$ para Q2; lo que deja como rendimiento en peso para la quitina $23 \%$ para Q1 y 19\% para Q2. Finalmente, las etapas empleadas en el proceso de desacetilación arrojaron un valor de pérdida de masa de alrededor de 5\% por etapa para Q1, mientras que para Q2 fue de alrededor de $1.5 \%$ por etapa, dejando como resultado un rendimiento de quitosano en relación a la materia prima de $15.96 \%$ para Q1 y de $16.31 \%$ para Q2 (base seca) lo que concuerda con estudios relacionados al contenido típico de componentes de la cáscara del camarón (Agulló et al., 2004).

Las características principales del quitosano, como son el grado de desacetilación \%DD (87\% Q1 y $94 \%$ Q2) y el peso molecular promedio $\left(5.2 \times 10^{5}\right.$ Q1 y $5.4 \times 10^{5}$ Q2), presentaron valores característicos de quitosanos comercializados en la actualidad, lo que permite establecer su calidad. Adicionalmente, el proceso utilizado para obtener estos dos tipos de quitosano variaron únicamente en la proporción solvente:soluto en cada proceso establecido en la Figura 1, valor que fue mayor para el quitosano Q2 que para Q1. En consecuencia, al usar una mayor proporción solvente:soluto, se utilizaron menos etapas de desacetilación para Q2 en comparación con Q1.

El modelo de correlación estadístico, presentado en la Figura 5 y detallado en la Tabla 5, presenta evidencia estadísticamente significativa del efecto que tienen los factores estudiados, esto es, $\mathrm{pH}$ $(P<0.0001)$, tipo de quitosano $(P<0.0001)$ y método de agitación $(P<0.0032)$, en la variable de respuesta, que es el porcentaje de remoción de turbidez. Seguidamente, con el uso de los datos estadísticos se trató la muestra contaminada Mc2 y se observó la formación de flóculos aglomerados en una fase liviana, atribuyendo este comportamiento a la gran cantidad de hidrocarburos (TPH) presentes en la muestra como se muestra en la imagen D de la Figura 2 y en la imagen B de la Figura 6. Finalmente, se obtuvo una remoción positiva para la turbidez 98.19\%, DQO 78.17\%, color $91.45 \%$, TPH 99.09\%, existiendo una presencia no detectable, por los equipos acreditados del Grupo Químico Marcos, de Bario, Cromo, Plomo y Vanadio (Tabla 6).

\section{Conclusiones y Recomendaciones}

El residuo del empacado de camarón para exportación, así como el proveniente de cualquier otra actividad relacionada, puede ser utilizado en la obtención de quitina y quitosano de muy buena calidad como se presentó en este estudio, dándole un valor comercial a un residuo que constituye un agente contaminante en las zonas de producción del crustáceo en nuestro país. 
La metodología utilizada para el uso de quitosano como biocoagulante en la remoción de contaminates de aguas residuales de la comercialización de hidrocarburos, resultó en la determinación del efecto estadístico que tienen los factores estudiados y como consecuencia un modelo estadístico que permitió conocer la mejor combinación aplicación de estos factores para alcanzar la mayor remoción de turbiedad (98\%) posible y consigo la remoción de Hidrocarburos Totales de Petróleo (99\%) presentes en este tipo de aguas residuales.

Se debería establecer un estudio en cuanto a la biorremediación de los lodos formados en el tratamiento del tipo de aguas residuales presentados. En este sentido, un mayor esfuerzo debe destinarse al desarrollo de sustancias de origen natural que puedan utilizarse en el tratamiento de aguas residuales y de consumo con el fin de preservar y garantizar la salud de los seres vivos además de la fauna y flora de nuestro ambiente.

\section{Bibliografía}

Abu Hassan, M. A. a. T., Pei Li and Noor, Zainura Zainon. (2009). Coagulation and flocculation treatment of wastewater in textile industry using chitosan. Journal of Chemical and Natural Resources Engineering., 4(1), pp. 43-53.

Agulló, E., Tapia, C., Peniche, C., Heras, Á., Argüelles, W., \& Nakamatsu, J. (2004). Fuentes y procesos de obtención. In A. P. d. Abram (Ed.), Quitina y Quitosano: obtención, caracterización y aplicaciones (Primera edición ed., pp. 113). Lima, Perú: Fondo Editorial de la Pontificia Universidad Católica del Perú.

Banco Central del Ecuador. (2014). La Economía Ecuatoriana Cerró El Año Con Un Saldo Neto De Usd 1.845,9 Millones, Correspondiente A 2.0\% Del Pib. Retrieved from http://www.bce.fin.ec/index.php/boletines-de-prensa-archivo/item/625-la-econom\%C3\%ADaecuatoriana-tuvo-un-crecimiento-anual-de-45-en-2013.

Caldera, Y., Clavel, N., Briceño, D., Nava, A., Gutiérrez, E., \& Mármol, Z. (2009). Quitosano como coagulante durante el tratamiento de aguas de producción de petróleo. Boletín del Centro de Investigaciones biológicas, 43, 541-555.

Czechowska-Biskup, R., Jarosińska, D., Rokita, B., Ulański, P., \& Rosiak, J. M. (2012). Determination of degree of deacetylation of chitosan-comparison of methods. Progress on Chemistry and Application of Chitin and Its Derivatives, 17, 5-20.

Da Ros, G. (1995). La contaminación de aguas en Ecuador: una aproximación económica: Editorial Abya Yala. 
Paz, N. d. I., Fernández, M., López, O. D., Nogueira, A., García, C. M., Pérez, D., . . Díaz, D. (2012). Optimización Del Proceso De Obtención De Quitosano Derivada De La Quitina De Langosta. Revista Iberoamericana de Polímeros, 13(3), 103-116.

Paz, N. d. I., Pérez, D., Fernández, M., López, O. D., Nogueira, A., Paneque, M. R., . . García, C. M. (2013). Evaluación Viscosimétrica Del Quitosano Derivado De La Quitina De Langosta. Revista Iberoamericana de Polímeros, 14(2), 84-91.

Pinelli Saavedra, A., Toledo Guillen, A. R., Esquerra Brauer, I. R., Luviano Silva, A. R., \& Higuera Ciapara, I. (1998). [Methods for extracting chitin from shrimp shell waste]. Arch Latinoam Nutr, 48(1), 58-61.

Reddy, C. M., Eglinton, T. I., Hounshell, A., White, H. K., Xu, L., Gaines, R. B., \& Frysinger, G. S. (2002). The West Falmouth oil spill after thirty years: the persistence of petroleum hydrocarbons in marsh sediments. Environ Sci Technol, 36(22), 4754-4760.

Renault, F., Sancey, B., Charles, J., Morin-Crini, N., Badot, P.-M., Winterton, P., \& Crini, G. (2009). Chitosan flocculation of cardboard-mill secondary biological wastewater. Chemical Engineering Journal, 155(3), 775-783. doi:10.1016/j.cej.2009.09.023

Roberts, G. A. F., \& Domszy, J. G. (1982). Determination of the viscometric constants for chitosan. International Journal of Biological Macromolecules, 4(6), 374-377. doi:http://dx.doi.org/10.1016/0141-8130(82)90074-5

Rodríguez, Y. (2011). Eficiencia del quitosano como coagulante en el tratamiento del agua asociada a la producción de petróleo. (Master of Science Tesis de grado), Universidad del Zulia, Maracaibo, Venezuela. Retrieved from http://tesis.luz.edu.ve/tde_arquivos/80/TDE2012-04-24T10:02:04Z-2834/Publico/rodriguez_diaz_yim_james.pdf

Sastre, R. A. M., Ruiz, P. M., Guibal, E., \& Szygula, A. (2010). Aplicación del biopolímero quitosan en la eliminación del color de las aguas residuales mediante el proceso combinado de coagulación-floculación y adsorción: Google Patents.

Varela, M. (2011). Procesamiento de camarón para exportación (R6 y R2). Ecuador: Ministerio de Industrias y Productividad MIPYMES - FLACSO Ecuador. Retrieved from https://www.flacso.org.ec/portal/pnTemp/PageMaster/v1h0ohbg78sb6mncmkkr5w3mwji4ep. pdf.

Yaghobi, N., \& Mirzadeh, H. (2004). Enhancement of chitin's degree of deacetylation by multistage alkali treatments. Iranian Polymer Journal, 131-136. 